\title{
DNA Methylation Profiles and Their Diagnostic Utility in BC
}

\author{
Ming Shan, ${ }^{1}$ Lei Zhang, ${ }^{2}$ Yang Liu $\mathbb{D},{ }^{1}$ Chunyang Gao $\mathbb{D},{ }^{2}$ Wenli Kang $\mathbb{D},{ }^{3}$ Weiwei Yang, ${ }^{2}$ \\ Yan $\mathrm{He}\left(\mathbb{D},{ }^{2}\right.$ and Guoqiang Zhang $\mathbb{1}^{1}$ \\ ${ }^{1}$ Department of BC Surgery, Harbin Medical University Cancer Hospital, Harbin, China \\ ${ }^{2}$ Department of Pathology, Harbin Medical University, Harbin, China \\ ${ }^{3}$ Department of Oncology, General Hospital of HeiLongjiang Province Land Reclamation Headquarter, Harbin, China
}

Correspondence should be addressed to Yan He; he_yan419@163.com and Guoqiang Zhang; 6-fu@163.com

Received 24 October 2018; Revised 31 January 2019; Accepted 18 February 2019; Published 6 May 2019

Academic Editor: Samanta Salvi

Copyright (c) 2019 Ming Shan et al. This is an open access article distributed under the Creative Commons Attribution License, which permits unrestricted use, distribution, and reproduction in any medium, provided the original work is properly cited.

Biomarkers, including DNA methylation, have shown a great potential for use in personalized medicine for BC and especially for the diagnosis of BC in developing countries. According to the bisulfite sequencing PCR in twelve specimens (BC and matched normal tissues), nine genetic probes were designed to detect the frequency of methylation of the promoters in a total of 302 paired cases of BC and matched normal breast tissues. Finally, a total of 900 serum samples were used to validate the use of these methylation biomarkers for clinical diagnosis of BC. A high frequency of promoter methylation of SFN, HOXA11, P16, RAR $\beta, P C D H G B 7, h M L H 1, W N T 5 a, H O X D 13$, and RASSF1a was observed in BC tissues. The methylation frequencies of HOXD13 and hMLH1 increased with the progression of BC. The methylation frequencies of HOXD13 and WNT5a were significantly higher in BC. We found that methylation modification-positive samples were most consistently associated with luminal BC. Finally, we confirmed that RASSF1a, P16, and PCDHGB7 displayed a significant sensitivity and specificity as diagnostic biomarkers for BC $(P<0.001)$, and a panel that combined these three genes displayed increased significance (AUC, $0.781 ; P<0.001)$. These data suggest that epigenetic markers in serum can potentially be used to diagnose BC. The identification of additional BC-specific methylated genes would improve the sensitivity and specificity of this approach. This study could also indicate that different molecular subtypes of $\mathrm{BC}$ are caused by distinct genetic and epigenetic mechanisms.

\section{Introduction}

Breast cancer (BC) is a complex and heterogeneous disease and a leading cause of death among women. Some regional surveys have indicated that the incidence of $\mathrm{BC}$ is also rising in Chinese women [1]. Approximately, the incidence of BC was $26.86 \%$ and the mortality of BC was $6.95 \%$ among Chinese women in 2015. High incidence is concentrated between 45 and 59 years old [2].

Carcinogenesis is a multistep process that results from the accumulation of genetic and epigenetic alterations [3]. Recent reviews have emphasized that epigenetic abnormalities might play an influential role in the earliest steps of cancer initiation and the progression of malignancies [4, 5], especially because the methylation of a normal allele can serve as a "second hit" that leads to gene inactivation when paired with mutations in the opposite allele [6].
Approximately $40-50 \%$ of human genes have CpG islands (CGIs) located in or near the promoter and/or first exon, and the methylation of these CGIs is critical to regulate the expression of these genes [7]. Alterations in the methylation status of DNA are among the most frequent molecular changes that are associated with human cancers [8].

In BC, many studies have investigated methylation patterns as potential biomarkers for detection, subtype classification, risk stratification, monitoring prognoses, and predicting susceptibility or responsiveness to a particular therapy $[9,10]$. However, in spite of the promise of such biomarkers, several barriers continue to prevent rapid progress toward using these markers in clinical applications. Major limitations to the further development of these markers in clinical applications might be that many studies have focused on investigating the methylation patterns in circulating free DNA (cfDNA) derived from the serum of healthy women 
and women with $\mathrm{BC}$, but studies rarely use benign breast tissues as the control to identify the potential clinical applications of using serum DNA methylation as a biomarker. In addition, these studies have investigated fewer BC and matched control specimens, and validation with larger patient cohorts has not been pursued [11, 12]. Other limitations include the utilization of different technologies by different laboratories, resulting in a range of detection sensitivities, a varying emphasis on quantitation, and the utilization of different sample processing methodologies and different reference materials as controls during analysis of hypermethylation degrees when using the same technology [13]. We therefore investigated a new diagnostic tool for BC and sought to overcome these barriers by using methylation genes as cancer biomarkers. We determined whether these genes could also be useful markers for predicting a prognosis in $\mathrm{BC}$ patients according to the progression of the cancer. These were ductal carcinoma in situ (DCIS), invasive ductal carcinoma (IDC), and invasive ductal carcinoma plus lymph metastasis (IDC-L).

In the present study, twelve candidate markers for $\mathrm{BC}$

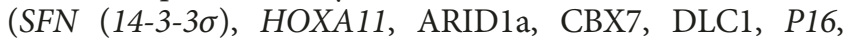
RAR $\beta, \quad P C D H G B 7, h M L H 1, \quad W N T 5 a, H O X D 13$, and RASSF1a) were studied with regard to their detection in BC tissues and matched serum samples. These genes have previously been shown to undergo cancer-specific methylation in breast tissues in the TCGA database [14] and other reports of clinical or fundamental studies [15-20]. These markers are representatives of a variety of cellular pathways, including DNA binding, cell cycle/checkpoint control, developmental regulation, chromatin binding, cell adherence, and cytokine activity. In addition, HOXA11, HOXD13, and PCDHGB7 were confirmed as early methylated genes when human mammary epithelial cells (HMEC) converted into cancer cells in our previous study and in other studies [19]. We examined the methylation status of the promoters of these candidate genes in two independent sets (test and validation) using a total of 302 paired tissue/normal samples. A matched serum detection assay of the validation set $(n=194)$ was then used to confirm the results obtained for the top hypermethylated genes from both the test and the validation sets, to show the reliable cfDNA methylation markers that diagnose BC. Finally, we identified the methylation biomarkers that best differentiated BC in a total of 900 serum samples that included samples from $300 \mathrm{BC}$ patients, 300 patients with benign breast diseases, and 300 healthy women.

\section{Materials and Methods}

2.1. Patients, Sample Collection, and DNA Extraction. All individuals signed surgical or clinical research consent forms allowing tissue and serum collection in accordance with the regulations approved by the IRB Committee of Harbin Medical University. This research was completed in compliance with the Helsinki Declaration. A brief outline of the study process is shown in Supplemental Figure 1. The study extended over biomarker development phases 1 and 2, which were based on Early Detection Research Network (ERDN) guidelines $[13,21]$. All the tissue and serum samples were obtained from patients and healthy persons undergoing physical examination at the Affiliated Tumor Hospital of Harbin Medical University, Harbin, China, from 2014 to 2017. Fresh-frozen specimens derived from cancerous and self-pair normal breast tissues $(\geq 5 \mathrm{~cm}$ distant from the tumor tissue) were obtained from patients who underwent a mastectomy for BC. The benign breast diseases included fibroadenoma, benign phyllodes tumors, mastopathy, papilloma, duct ectasia, and hamartoma (Supplemental Table 3). Healthy serum samples were acquired from the Affiliated Tumor Prevention and Treatment Institution of Harbin Medical University. All $\mathrm{H} \& \mathrm{E}$ slides were reviewed by two independent pathologists to determine the integrity of the tumor specimen (tumor content of $>70 \%$ ) and the normal tissue blocks, in which no tumor cells were observed.

All samples were classified as one of four types of primary BC lesions: (1) pure DCIS, 100 cases; (2) IDC, 100 cases; and (3) IDC-L, 102 cases. Genomic DNA was isolated from freshfrozen primary breast tumors and matched normal breast tissues. Samples were pretreated with proteinase K $(20 \mathrm{mg} / \mathrm{mL})$ at $55^{\circ} \mathrm{C}$ overnight and DNA was then extracted using an AxyPrep $^{\mathrm{TM}}$ Multisource Genomic DNA Miniprep Kit (Axygen Scientific Inc., CA, USA). Approximately $5 \mathrm{~mL}$ of peripheral blood was drawn into a blood collection tube prior to a physical examination or surgery, and all samples were transferred to the study laboratory within 4 hours of collection for processing. Circulating free DNA (cfDNA) was obtained from $1 \mathrm{~mL}$ of serum using a QIAamp Circulating Nucleic Acid Kit [22] (Qiagen, Hilden, Germany) according to the manufacturer's instructions.

2.2. Immunohistochemistry and Molecular Subtypes. The monoclonal ER antibody was obtained from Ventana (cata$\log$ no. 760-2596). The monoclonal PR antibody was obtained from Dako (catalog no. M3569). Nuclear labeling for ER positivity or PR positivity was required in greater than $1 \%$ of cells [23]. HER-2 IHC was performed using the Dako HercepTest kit according to the manufacturer's protocol. Cases were scored using the established criteria as $0,1+, 2+$, or $3+$. Fluorescence in situ hybridization analysis to determine Her-2 amplification was performed on all 2+ (equivocal) cases using the PathVysion kit (Des Plaines, IL). To qualify as Her-2 positive in this study, a case had to demonstrate either a 3+ IHC score or a Her-2 fluorescence in situ hybridization amplification ratio of greater than 2.2. Cases were categorized into one of four categories based upon accepted and previously validated IHC surrogate profiles of BC. Luminal A tumors were immunoreactive for ER and/or PR and negative for Her-2 or low proliferation. Tissue that was $\mathrm{ER}+$ and/or PR+, either Her2+ and/or highly proliferative, was considered luminal B tumors. The Her-2 subtype was defined as ER-, PR-, and Her2+. Basal-like tumor was the most controversial type. On the basis of the published criteria, basal-like cases were defined as tissues with a triple-negative phenotype (ER-/PR-/Her2-). We therefore used triple-negative BC (TNBC) instead.

IHC for p53 (Ventana, monoclonal antibody, catalog no. 760-2542) and Ki-67 (Ventana, monoclonal antibody, 
catalog no. M7240) only showed nuclear labeling. For p53, a labeling score indicating that $>30 \%$ of the nuclei were labeled was defined as aberrant overexpression (which correlates well but not perfectly with the presence of p53 mutation) [24]. The Ki67 cut-off point was $20 \%$, and this was used to designate a tumor as highly proliferative when assigning samples to subtype groups [25].

\subsection{Bisulfite Treatment, Sequencing, and MethyLight. Bisul-} fite conversion of genomic DNA was performed using an EZ DNA Methylation kit (Zymo Research, Orange, CA, USA) according to the manufacturer's instructions. Converted DNA was amplified using PCR as described in Supplemental Excel 1. For each BSP, ten positive clones were sequenced in both directions by the Life Technologies Lab (Invitrogen, Burlington, ON, CA).

According to the results of BSP sequencing, we selected the probable promoter $\mathrm{CpG}$ islands that contained the methylated variant sites to design probes for each gene (Supplemental Figure 1). A detailed list of the nucleotide sequences corresponding to the MethyLight primers and probes in the promoter or $5^{\prime}$ end region of all analyzed loci is provided in Supplemental Excel 2. TaqMan MGB (Applied Biosystems, Foster City, CA, USA) PCR was performed using primers specific for the bisulfite-converted methylated sequence of a particular locus. Globin reference primers were used separately. The TaqMan MGB probes showed a significant improvement in assay specificity, and their smaller size allowed for a more flexible assay design.

MethyLight is highly specific, sensitive, and reproducible. It can also rapidly detect biologically relevant information in patient samples. MethyLight is a PCR-based method that requires only very small amounts of DNA of modest quality, and this makes it compatible with small biopsies and paraffin-embedded tissues [26]. MethyLight could therefore be a utility tool for use in clinical applications [13]. The majority of studies that have used percentage of methylated reference (PMR) as a method for evaluating methylation have reported positive results. But the cut-off value for PMR varies when used with MethyLight in different studies [15, 27-29]. This is likely the result of not using self-matched normal tissue as a control in studies that instead use SssI-treated human peripheral white blood cell DNA from the same person or from healthy people as the control. This comparison may not accurately reflect positive methylation cases, because methylation modification is influenced by many factors, including lifestyle, environmental exposure, ethnicity, age, and tissue heterogeneity [26, 30]. In this study, we compared BC tissue to matched normal breast tissue (distant from tumor mass $\geq 5 \mathrm{~cm}$ ) from the same person, and the percentage of samples that were methylated at a specific locus was statistically calculated using the $2^{-\Delta \Delta \mathrm{Ct}}$ method, where $\Delta \Delta \mathrm{Ct}=\left(\mathrm{CT}_{\text {Target gene }}-\mathrm{CT}_{\text {Reference }}\right)$ sample - $\left(\mathrm{CT}_{\text {Target gene }}-\mathrm{CT}_{\text {Reference }}\right)$ control (matched normal tissue from the same patient) [31]. All samples were assayed in duplicate, and to validate the results of the $2^{-\Delta \Delta \mathrm{Ct}}$ method, the amplification efficiencies of the test genes and a reference gene, Globin, were examined using serial dilutions of DNA over a 100-fold range and using gene-specific primers for each gene and Globin. The $\Delta \mathrm{Ct}\left(\mathrm{CT}_{\text {Target gene }}-\mathrm{CT}_{\text {Reference }}\right)$ was calculated for each DNA dilution, and a plot of the log DNA dilution vs. $\Delta \mathrm{Ct}$ was constructed. A cut-off value of $\geq 1.5[32,33]$ (allelic gene methylation) was determined to indicate a positive result. The analysis of cfDNA methylation frequency was also performed using the MethyLight method. We used $2^{-\Delta \mathrm{Ct}}\left(\Delta \mathrm{Ct}\right.$ was calculated as $\mathrm{CT}_{\text {Targetgene }}-$ $\left.\mathrm{CT}_{\text {Reference }}\right)$ in a ROC curve analysis to determine both sensitivity and specificity in comparison of results between BC and control samples (including healthy women and patients with benign breast diseases).

2.4. Statistical Analysis. Data were analyzed using Student's $t$-tests, Fisher's exact tests, Kruskal-Wallis $H$, ROC curve analyses, and Mann-Whitney $U$ tests. All tests were performed using SPSS 17.0. A $P$ value of $<0.05$ was considered significant.

\section{Results}

3.1. Prescreening of the Promoter CpG Islands of Candidate Genes to Select Methylation Targets Using BSP. From the TCGA database, gene methylation biomarkers identified for the diagnosis of other tumors in the previous studies, and the distinct methylation genes identified during the conversion from human normal mammary epithelial cells to $\mathrm{BC}$

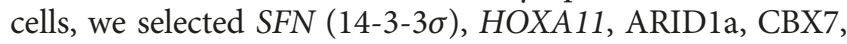
DLC1, P16, RAR $\beta$, PCDHGB7, hMLH1, WNT5a, HOXD13, and RASSF1a, which represent a variety of different pathways that are involved in cancer (Supplemental Excel 3). Initially, we evaluated the $\mathrm{CpG}$ islands of all of these genes [34], and the highly dense regions containing the CpG sites in the $\mathrm{CpG}$ islands were sequenced using BSP in six paired cases of BC tissues and matched normal breast tissues (Supplemental Figure 2). DLC1 was eliminated because the highly dense $\mathrm{CpG}$ region could not be amplified using BSP. We then selected variant methylation sites that were methylated in at least half of the BC tissues and unmethylated in the matched breast normal tissues to design the probes for MethyLight (Supplemental Table 1).

3.2. Determination of Methylation Frequency in an Appropriate Gene Evaluation Set of Patients. Next, all residual tissue specimens were divided into two data sets: the test set (108 paired cases of BC tissues and matched normal breast tissues) and the validation set (194 paired cancer tissues and matched normal tissues). All of these samples were obtained from BC patients aged 40-60 years old to rule out the effect of age on DNA methylation. The other clinicopathological factors, including the pathological type, histological grade, BMI, and tumor size, were not different $(P>0.05$, Supplemental Table 2$)$ between the test set and the validation set.

We investigated the methylation frequency of eleven genes between $\mathrm{BC}$ tissues and matched normal breast tissues in the test set using MethyLight. Significantly high methylation frequencies were detected for nine genes in $\mathrm{BC}$ tissues from the test set. Moreover, we next confirmed these results 
using the same nine methylation probes in the validation set, and we found that they also displayed the high methylation frequencies (Supplemental Table 4). In conclusion, a total of nine methylated genes, including SFN, HOXA11, $P 16, R A R \beta$, PCDHGB7, hMLH1, WNT5a, HOXD13, and RASSF1a, were methylated with significantly higher frequency in BC tissues than in matched normal breast tissues from 302 BC patients. The average methylation frequencies for all of the genes in the BC tissue group are shown in Supplemental Table 5. Among all of these markers, PCDHGB7 was most often methylated (78.81\%), whereas the lowest methylation frequency was observed for WNT5a (28.48\%).

3.3. Methylation Frequency during the Progression of BC. We categorized all of the malignant samples into four groups according to the histopathology of $\mathrm{BC}$, including DCIS, IDC, and IDC-L. The results of the methylation frequency analysis for all nine genes is illustrated in Table 1. All of the genes displayed widespread aberrant promoter $\mathrm{CpG}$ island methylation. The frequency of HOXD13 and hMLH1 methylation significantly increased with the progression of the disease from in situ to invasive cancer $(P<0.001$ and $P<0.05$, Figure 1), but there was no significant difference between IDC and IDC-L.

\subsection{Methylation Profiles Associated with Molecular Subtypes} and Clinicopathological Features. In each group (DCIS, IDC, and IDC-L), we classified three subgroups according to the coexistence of methylation between genes: coexistence of one to three methylation genes, four to six methylation genes, or seven to nine methylation genes (Supplemental Table 6). We found that the category with the fewest genes was the group indicating the coexistence of seven to nine methylated genes in the DCIS group (5\%). To exclude contingency and on the basis of the prior studies [35], we determined that samples in which at least three genes were simultaneously methylated were likely to be affected by epigenetic modifications (especially DNA methylation modifications) and we named these "methylation modification-positive samples." Next, we analyzed the specimens that clustered with the BC molecular subtypes in different groups (Figure 2). We found that methylation modification-positive samples were consistently the luminal type of BC (Figure 3 ) in the DCIS, IDC, and IDC-L groups.

\subsection{Evaluation of the Consistency of Methylation Frequency} between BC Tissues and Matched Serum and Determination of the Best-Performing Methylation Probes in BC Diagnoses. Based on the above data, we have shown that gene methylation frequencies are significantly higher in BC tissues. Next, we considered whether methylation can be used as a diagnostic marker of BC. We used specific probes to assess the methylation of nine genes in matched serum samples in the validation set using MethyLight. The majority of the genes that were methylated in the $\mathrm{BC}$ tissue were also methylated in the matched cfDNA obtained from serum (in the gene methylation-positive tissues). The same genes displayed higher average frequencies in the matched serum, including PCDHGB7, P16, and RASSF1a (Table 2). Meanwhile, the
TABLE 1: Methylation frequencies for the nine genes in breast cancer patients.

\begin{tabular}{lccc}
\hline \multirow{2}{*}{ Methylated gene } & \multicolumn{3}{c}{ Breast cancer tissue (302 cases) } \\
& DCIS & IDC & IDC_L \\
\hline SFN & 25.67 & 27.35 & 36.67 \\
HOXA11 & 38.23 & 40.67 & 45.34 \\
P16 & 37.45 & 44.5 & 42.11 \\
RASSF1a & 64.45 & 54.38 & 68.45 \\
PCDHGB7 & 75.56 & 83.45 & 76.45 \\
hMLH1 & $\mathbf{2 3 . 5 5}$ & $\mathbf{4 0 . 5 7}$ & $\mathbf{4 7 . 4 3}$ \\
Wnt5a & $\mathbf{3 1 . 3 4}$ & $\mathbf{3 4 . 5 6}$ & $\mathbf{3 6 . 4 5}$ \\
HOXD13 & $\mathbf{3 2 . 3 4}$ & $\mathbf{6 5 . 3 3}$ & $\mathbf{6 6 . 7 8}$ \\
RAR $\beta$ & 43.45 & 24.78 & 34.68 \\
\hline
\end{tabular}

frequency of HOXA11 or WNT5a methylation was low in serum, even though the frequency of methylation of these markers in the matched BC tissues was high. In addition, HOXD13 was only methylated in 1 cfDNA sample, and $R A R \beta$ was only methylated in 3 samples of cfDNA. The methylation frequency of $h M L H 1$ was $33.33 \%$ in serum and $35.57 \%$ in tissues. Finally, the observation that frequencies increased along with the progression of $\mathrm{BC}$, as observed in tissues and illustrated in Figure 3, did not recur in the serum methylation study.

According to the frequent study of methylation genes in breast cancer tissues and serum samples, we selected genes that had a higher methylation frequency in both the breast cancer tissues and the matched serum samples to explore the clinical utility of using such methylation biomarkers to diagnose breast cancer. PCDHGB7, P16, and RASSF1a (although the methylation frequency of RASSF1a was lower in the serum in this study, it was generally high in breast cancer tissues) were selected. We used an expanded set of serum samples that included 300 breast cancer samples, 300 samples from age-matched healthy controls, and 300 samples from age-matched patients with benign breast diseases. In the ROC curves corresponding to the three analyzed genes (Figure 4(a)), RASSF1a showed a sensitivity of 75\%, a specificity of $62.5 \%$, and an area under the curve (AUC) of 0.682 (95\% CI, 0.645 to $0.719, P<0.001$ ). The sensitivity and specificity of $P 16$ were $75 \%$ and $64.33 \%$, respectively, and the AUC was 0.687 (95\% CI, 0.650 to $0.724, P<0.001$ ). PCDHGB7 showed the highest sensitivity (84.33\%). This is in accordance with our results showing that this marker showed the highest methylation frequency in breast cancer tissues (Supplemental Table 5). However, the specificity of serum PCDHGB7 was not very high (60.33\%), and the AUC for this marker was 0.660 (95\% CI, 0.630 to 0.678 , $P<0.001)$. Next, we performed an ROC curve analysis for a three-gene panel to determine its sensitivity and specificity for diagnosing breast cancer (Figure 4(b)). According to this analysis, the three-gene panel discriminated between breast cancer patients and controls with a sensitivity of $82.67 \%$ and a specificity of $77.83 \%$ (AUC, $0.781 ; 95 \%$ CI, 0.757 to $0.796, P<0.001)$. This combination of three 


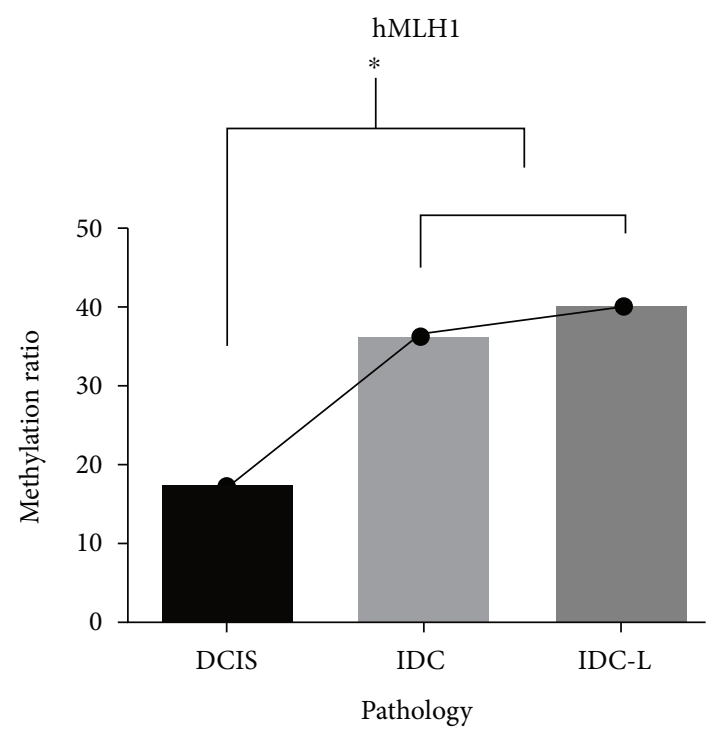

(a)

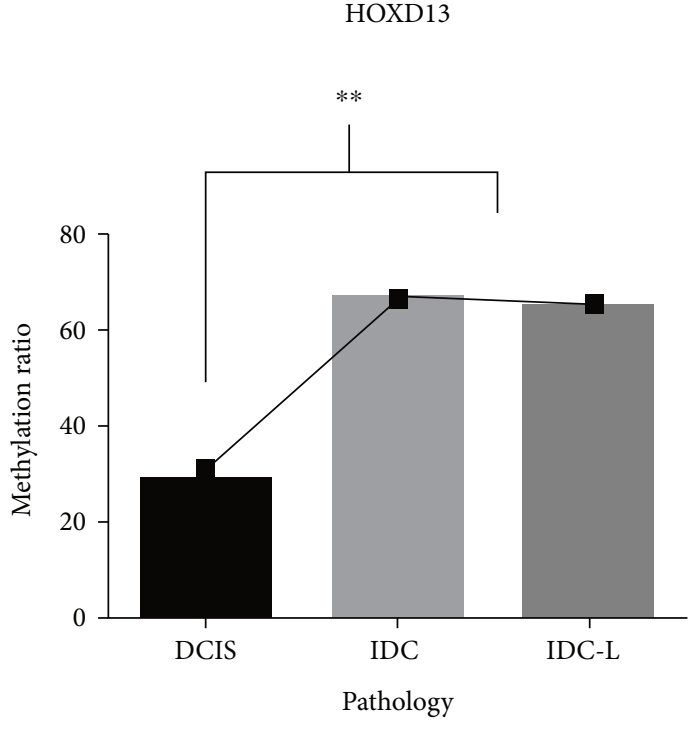

(b)

Figure 1

different methylation markers maximized their significance in the clinical diagnosis of breast cancer.

\section{Discussion}

The recent report has indicated that the incidence of $\mathrm{BC}$ in developing countries is stably increasing [35]. However, distinctive features related to $\mathrm{BC}$ in Asian women relative to women of predominantly European ancestry include Asian women who have larger tumors and a more advanced tumor stage at diagnosis, and these characters are associated with delayed diagnosis or more aggressive disease [1, 22, 36]. Approximately $75 \%$ of cases in Asian women are diagnosed at late and untreatable stages (clinical stages III and IV) $[37,38]$. This may be due to lack of awareness, limited healthcare infrastructure, inadequate manpower, and the uneven distribution of resources. Mammography has been the "gold standard" for BC detection for decades, and it is commonly applied in western countries. However, it is not suitable for use in developing countries as a diagnostic tool for BC because of limitations, including age, the density of breast tissues, socioeconomic factors, and medical resources [34, 39]. It is therefore necessary to explore efficacious, economical, convenient, and practical diagnostic methods that are suitable for use in developing countries.

In this study, we screened the methylation status of nine genes belonging to different molecular pathways in different pathological BC and matched normal tissues. This could raise the accuracy of such biomarkers for determining a diagnosis or prognosis in BC $[40,41]$. Although RASSF1a, RAR $\beta$, $S F N, h M L H 1$, and $P 16$ have been widely detected in different studies $[18,30,42,43]$ and in different people $[27,44,45]$, we report important data regarding the frequency of the methylation of genes in BC. We found that HOXA11, PCDHGB7, and HOXD13 are also highly methylated in BC tissues, which was rarely reported in the prior studies, especially in DCIS.
Furthermore, we show that not all of the genes that are methylated in tumor tissues are also highly methylated in serum cfDNA, as the case for HOXD13 and HOXA11. Although knowledge of the underlying mechanisms involved in determining the levels of these genes in circulating DNA is still limited [46], some evidence suggests that cfDNA is released from tumors as a glyconucleoprotein complex, which might protect it from degradation by nucleases [47]. It remains unclear whether the release of tumor DNA into serum is associated with tumor necrosis, apoptotic cell death, or other selective cellular processes. Because it is presumably shed from the original primary tumor, cfDNA might be fragmented and the quantity of cfDNA is greatly reduced. It has therefore been suggested that the clinical utility of using methylated biomarkers to diagnose $\mathrm{BC}$ must be confirmed in the serum and not just in $\mathrm{BC}$ tissues. For diagnostic biomarkers of $\mathrm{BC}$, we selected RASSF1a, which is widely used as a methylation biomarker for diagnosing $\mathrm{BC}$ in western countries, and P16 and PCDHGB7 because they are highly methylated in both BC tissues and serum. RASSF1a and P16 displayed a significant utility for diagnosing BC, as found in the prior studies. However, the sensitivity and specificity observed in this study were different from that found in the previous studies [16, 48, 49]. The most important reason for this discrepancy could be that we added benign controls in this study, and this may have reduced the sensitivity and specificity of these markers in contrast with results that compare only BC and healthy samples. Other reasons could be differences in the race of the sample population or their environment, difference in methodology, and differences in the targets being investigated. Hence, in future research aimed at investigating methylation to determine diagnostic biomarkers in BC, we strongly suggest that samples should contain matched samples from patients with benign diseases. The methylation frequency observed in PCDHGB7 may be the first time that methylation has been detected in $\mathrm{BC}$ tissue 


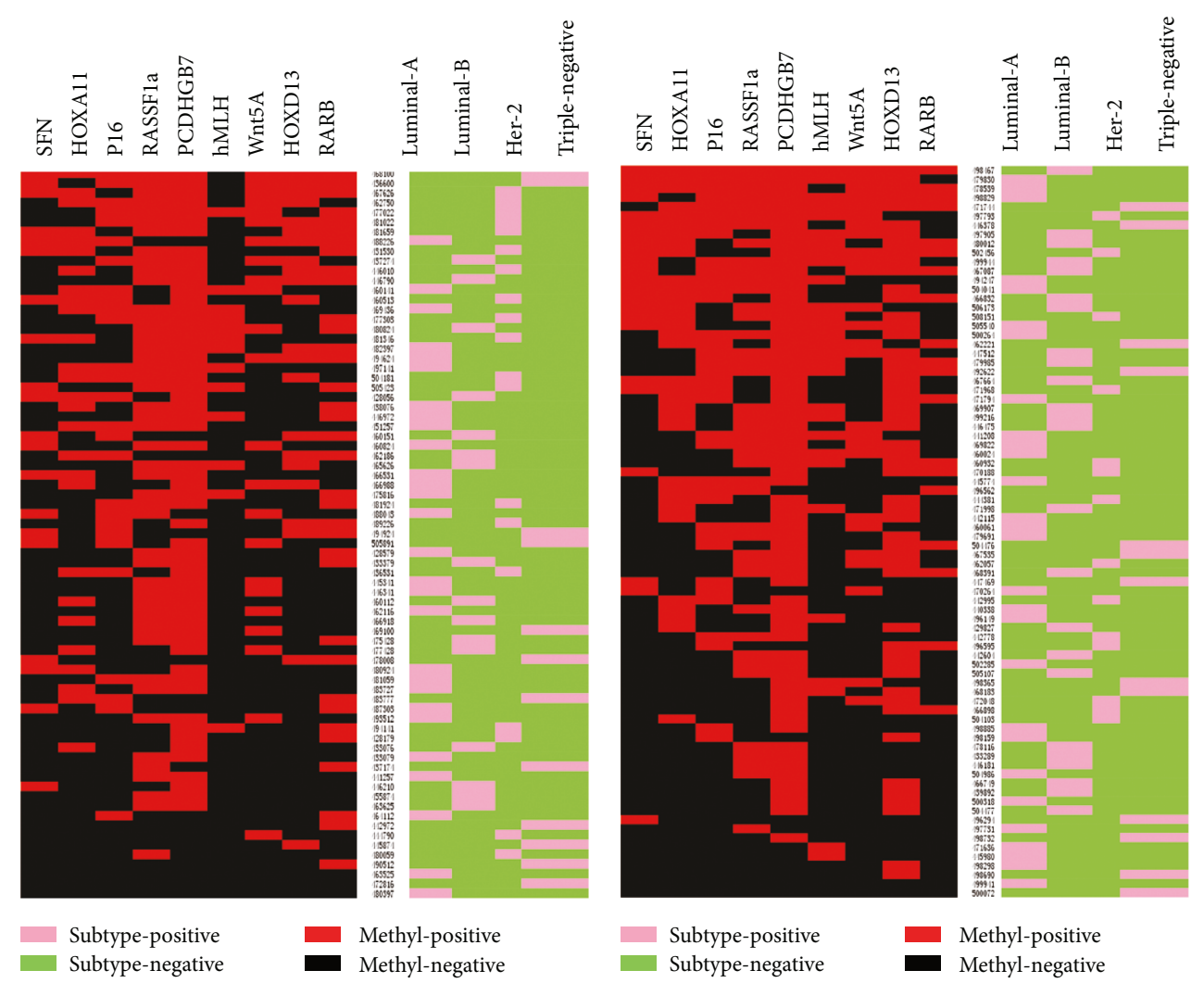

(a)

(b)

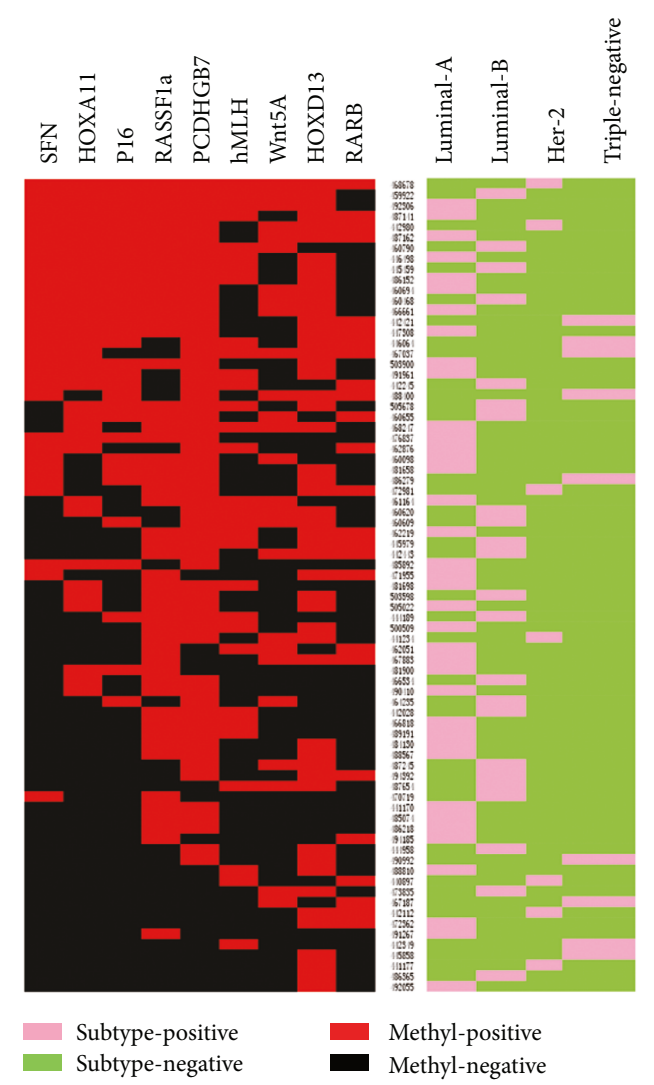

(c)

Figure 2 
DCIS

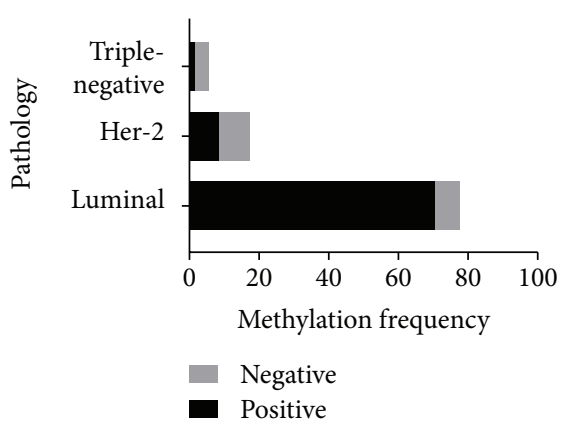

(a)
IDC

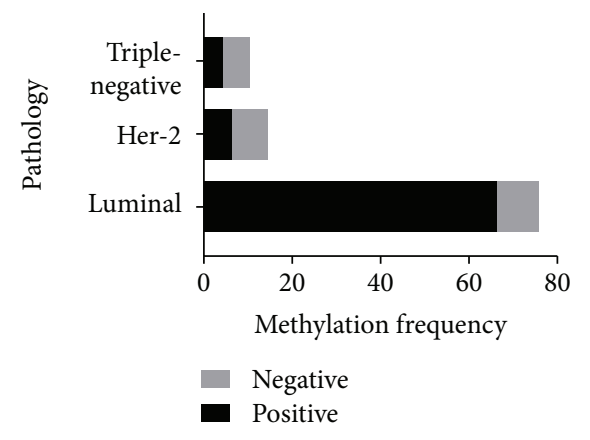

(b)
IDC-L

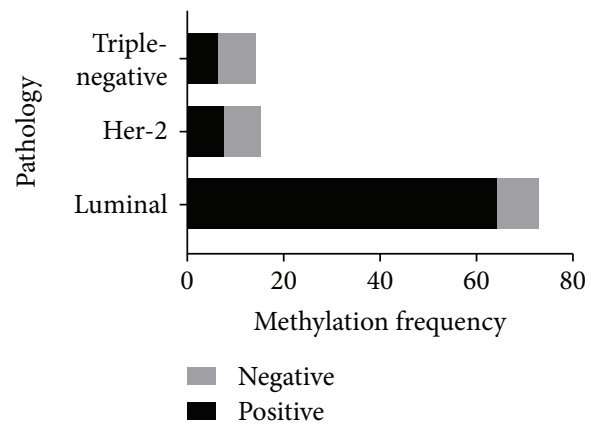

(c)

Figure 3

TABLE 2: Methylation frequencies of the same genes in the matched serum and methylation-positive BC tissues.

\begin{tabular}{|c|c|c|c|c|c|c|}
\hline \multirow{2}{*}{ Methylated gene } & \multicolumn{2}{|c|}{$\begin{array}{c}\text { Breast cancer tissue } \\
\text { (validation test, } 194 \text { cases) }\end{array}$} & \multirow{2}{*}{ Methylation frequency (\%) } & \multicolumn{2}{|c|}{ Matched serum } & \multirow{2}{*}{ Methylation frequency (\%) } \\
\hline & Methyl case & Unmethyl case & & Methyl case & Unmethyl case & \\
\hline$S F N$ & 61 & 133 & 31.44 & 35 & 26 & 57.38 \\
\hline HOXA11 & 88 & 106 & 45.36 & 15 & 73 & 17.01 \\
\hline P16 & 86 & 108 & 44.33 & 48 & 38 & 55.81 \\
\hline RASSF1a & 117 & 77 & 60.31 & 43 & 74 & 36.75 \\
\hline PCDHGB7 & 156 & 38 & 80.41 & 79 & 77 & 50.64 \\
\hline$h M L H 1$ & 69 & 125 & 35.57 & 23 & 46 & 33.33 \\
\hline$W n t 5 a$ & 57 & 137 & 29.38 & 12 & 45 & 21.05 \\
\hline HOXD13 & 90 & 104 & 46.39 & 1 & 89 & 1.11 \\
\hline$R A R \beta$ & 71 & 123 & 36.6 & 3 & 68 & 4.23 \\
\hline
\end{tabular}

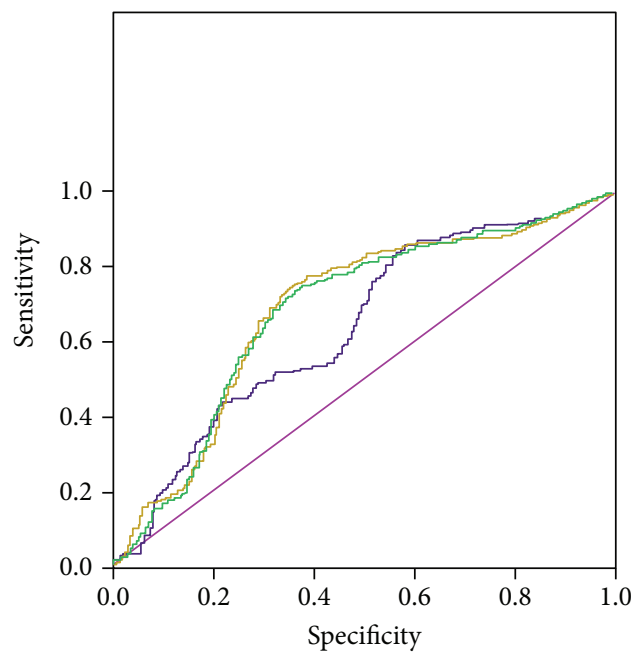

Source of the curve

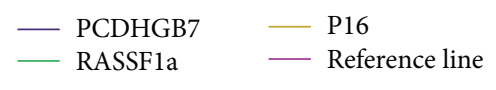

(a)

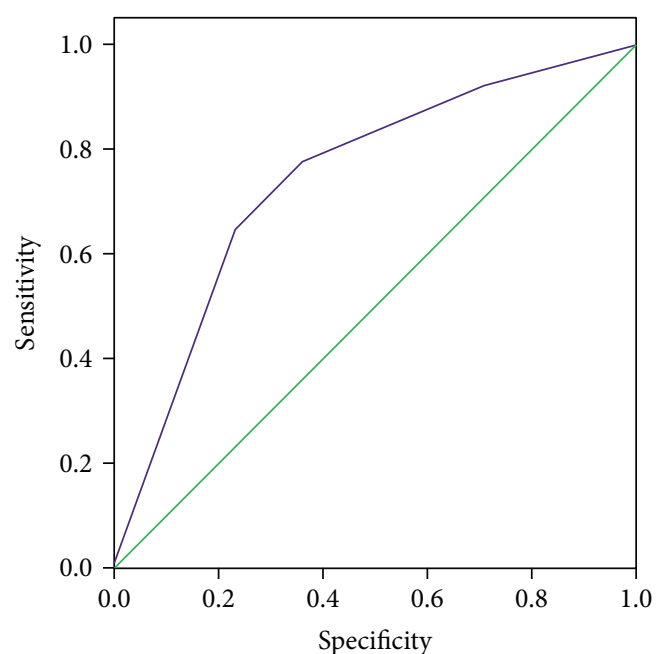

Source of the curve

PCDHGB7+RASSF1a+P16
Reference line

(b)

Figure 4 
and serum and found to be an effective methylation biomarker for the diagnosis of $\mathrm{BC}$. In addition, we also investigated the mRNA expression of PCDHGB7 in BC tissues to confirm its methylation in $\mathrm{BC}$. The results showed that $P C D H G B 7$ was expressed at low levels in most BC tissues (approximately $80 \%$, Supplemental Figure 3). Finally, we confirmed that a panel of methylated biomarkers that included these three genes showed the best sensitivity and specificity for the diagnosis of $\mathrm{BC}$. Therefore, in future studies, we will add more effective methylation markers to increase the sensitivity and specificity of this panel for use in diagnosing BC.

Many studies have reported that the frequency of methylation should significantly increase in parallel with the progression of cancer $[19,33]$ and that methylation could therefore potentially be used as a predictor during the determination of a prognosis in BC. In our study, we showed that HOXD13 and $h M L H 1$, in BC tissue methylation detections, demonstrate this phenomenon. In particular, methylation of HOXD13, a member of the HOX family, significantly increased in parallel with the progression of BC (from in situ to metastasis). This example was also just reported by Gupta et al. [50] in Nature. However, this phenomenon did not reappear for either HOXD13 or hMLH1in the analysis of methylation in serum. As we discussed above, this reminds us that accordance in methylation profiles between tissues and the matched serum samples is not perfect, as reported by Korshunova et al. [51]. Many methylation biomarkers that have been detected in $\mathrm{BC}$ tissues in the prior reports may not be suitable for clinical diagnoses of BC unless they are also analyzed in the serum. We also found that the methylation frequency of $R A R \beta$ showed a significant variation: $43.45 \%$ in DCIS, $24.78 \%$ in IDC, and $34.68 \%$ in IDC-L, and these results followed the progression of $\mathrm{BC}$. This may be the result of chance, or cyclic methylation modification mechanisms might be present, as reported in the prior studies $[52,53]$, in the $R A R \beta$ promoter region, and these must be clarified in the future.

Finally, by performing an unsupervised clustering analysis of DCIS, IDC, and IDC-L, we found that luminal, Her-2, and triple-negative tumors had different methylation profiles. We synchronously clustered at least three methylation genes with different functions in a single specimen, relative to $\mathrm{BC}$ subtypes. The highest methylation frequencies were usually observed in luminal tumors. Her-2 and triplenegative $\mathrm{BC}$ samples displayed low methylation frequencies in general, and this result may be compatible with results indicating they have unstable and aberrant genomes, which may result from reduced transposon silencing. The association between methylated modification profiles and different subtypes has been mentioned in many previous investigations $[40,54,55]$ because it could indicate that different molecular subtypes of $\mathrm{BC}$ could be caused by distinct genetic and epigenetic mechanisms [56].

\section{Abbreviations}

GWAS: Genome-wide association studies

CGI: $\quad$ CpG islands
cfDNA: Circulating free DNA

DCIS: Ductal carcinoma in situ

IDC: Invasive ductal carcinoma

IDC-L: Invasive ductal carcinoma plus lymph metastasis

HMEC: Human mammary epithelial cells

ERDN: Early Detection Research Network

TNBC: Triple-negative BC.

\section{Data Availability}

(i) The following data (DATA TYPE) used to support the findings of this study are included within the article. The data are as follows: (1) methylation frequencies for the nine genes in sporadic and hereditary BC patients, (2) methylation frequencies of the same genes in the matched serum and methylation-positive BC tissues, (3) different methylation frequencies of HOXD13 and hMLH1 during the BC progression, (4) different methylation frequencies of HOXD13 and WNT5a between sporadic and hereditary BC, (5) DNAmethylated modification levels in different molecular subtypes of BC, and (6) ROC curve analysis of the three-gene methylation panel between $\mathrm{BC}$, age-matched healthy and benign samples. (ii) The following data (DATA TYPE) used to support the findings of this study are included within the supplementary information file(s). The data are as follows: (1) the BSP PCR reaction primers and the system used to analyze the twelve genes., (2) MethyLight primers, probes, and the system used to analyze the twelve genes, (3) the signaling pathways of the candidate genes, (4) primers, probe sequences, and tested methylation sites for all genes, (5) clinicopathologic parameters of patients with BC in the test and validation sets, (6) methylated frequencies of nine genes in $\mathrm{BC}$ tissues from the test and validation sets, (7) methylation frequencies for the nine genes in $\mathrm{BC}$ patients, (8) the list of coexisting methylated genes in specimens with different histopathological types in sporadic and hereditary BC, (9) BC specimens in which at least three genes were simultaneously methylated in different subtypes (DCIS, IDC, IDC_L, and HpBC), (10) overview of the analyzed procedure, (11) the BSP analysis and the methylated sites chosen for all of the genes, (12) cluster analysis of methylated genes by BC subtypes in different groups ((a) DCIS, (b) IDC, (c) IDC-L, and (d) HpBC), and (13) differential expression of PCDHGB7 between $\mathrm{BC}$ tissues and matched normal breast tissues.

\section{Conflicts of Interest}

The authors declare that they have no conflicts of interest.

\section{Authors' Contributions}

Ming Shan, Lei Zhang, and Yang Liu contributed equally to this work.

\section{Acknowledgments}

This study was supported by the Health and Family Planning Commission of Heilongjiang Province (2017-105), 
the Heilongjiang Province Science Fund (QC2015113), and the Heilongjiang Postdoctoral Foundation (LBH-Z16120, LBH-Z17209).

\section{Supplementary Materials}

Supplemental Table 1: primers, probe sequences, and tested methylation sites for all genes. Supplemental Table 2: clinicopathologic parameters of patients with breast cancer in the test and validation sets. Supplement Table 3: clinical pathological characteristics of benign and normal control cases. Supplemental Table 4: methylated frequencies of nine genes in breast cancer tissues from the test and validation sets. Supplemental Table 5: methylation frequencies for the nine genes in breast cancer patients. Supplemental Table 6: the list of coexisting methylated genes in specimens with different histopathological types in breast cancer. Supplemental Figure 1: overview of the analyzed procedure. Supplemental Figure 2: the BSP analysis and the methylated sites chosen for all of the genes. Supplemental Figure 3: differential expression of PCDHGB7 between BC tissues and matched normal breast tissues. (Supplementary Materials)

\section{References}

[1] Z. G. Yu, C. X. Jia, L. Y. Liu et al., "The prevalence and correlates of breast cancer among women in Eastern China," PLoS One, vol. 7, no. 6, article e37784, 2012.

[2] W. Chen, R. Zheng, P. D. Baade et al., "Cancer statistics in China, 2015," CA: A Cancer Journal for Clinicians, vol. 66, no. 2, pp. 115-132, 2016.

[3] R. Taby and J. P. J. Issa, "Cancer epigenetics," CA: A Cancer Journal for Clinicians, vol. 60, no. 6, pp. 376-392, 2010.

[4] S. B. Baylin and J. E. Ohm, "Epigenetic gene silencing in cancer - a mechanism for early oncogenic pathway addiction?," Nature Reviews Cancer, vol. 6, no. 2, pp. 107116, 2006.

[5] A. P. Feinberg, R. Ohlsson, and S. Henikoff, "The epigenetic progenitor origin of human cancer," Nature Reviews Genetics, vol. 7, no. 1, pp. 21-33, 2006.

[6] M. Esteller, M. F. Fraga, M. Guo et al., "DNA methylation patterns in hereditary human cancers mimic sporadic tumorigenesis," Human Molecular Genetics, vol. 10, no. 26, pp. 30013007, 2001.

[7] J. M. Craig and W. A. Bickmore, "The distribution of CpG islands in mammalian chromosomes," Nature Genetics, vol. 7, no. 3, pp. 376-382, 1994.

[8] M. Esteller, "Epigenetics in cancer," New England Journal of Medicine, vol. 358, no. 11, pp. 1148-1159, 2008.

[9] J. T. Ren, M. X. Wang, Y. Su, L. Y. Tang, and Z. F. Ren, "Decelerated DNA methylation age predicts poor prognosis of breast cancer," BMC Cancer, vol. 18, no. 1, p. 989, 2018.

[10] R. S. DeVaux and J. I. Herschkowitz, "Beyond DNA: the role of epigenetics in the premalignant progression of breast cancer," Journal of Mammary Gland Biology and Neoplasia, vol. 23, no. 4, pp. 223-235, 2018.

[11] P. W. Laird, "The power and the promise of DNA methylation markers," Nature Reviews Cancer, vol. 3, no. 4, pp. 253266, 2003.
[12] J. Brooks, P. Cairns, and A. Zeleniuch-Jacquotte, "Promoter methylation and the detection of breast cancer," Cancer Causes \& Control, vol. 20, no. 9, pp. 1539-1550, 2009.

[13] J. Kagan, S. Srivastava, P. E. Barker, S. A. Belinsky, and P. Cairns, "Towards clinical application of methylated DNA sequences as cancer biomarkers: a joint NCI's EDRN and NIST workshop on standards, methods, assays, reagents and tools," Cancer Research, vol. 67, no. 10, pp. 4545-4549, 2007.

[14] "Targeting molecular tumor types," Nature Genetics, vol. 45, no. 10, p. 1103, 2013.

[15] M. Esteller, "Cancer epigenomics: DNA methylomes and histone-modification maps," Nature Reviews Genetics, vol. 8, no. 4, pp. 286-298, 2007.

[16] L. Van De Voorde, R. Speeckaert, D. Van Gestel et al., "DNA methylation-based biomarkers in serum of patients with breast cancer," Mutation Research/Reviews in Mutation Research, vol. 751, no. 2, pp. 304-325, 2012.

[17] H. M. Byun, K. D. Siegmund, F. Pan et al., "Epigenetic profiling of somatic tissues from human autopsy specimens identifies tissue- and individual-specific DNA methylation patterns," Human Molecular Genetics, vol. 18, no. 24, pp. 4808-4817, 2009.

[18] K. P. M. Suijkerbuijk, P. J. van Diest, and E. van der Wall, "Improving early breast cancer detection: focus on methylation," Annals of Oncology, vol. 22, no. 1, pp. 24-29, 2011.

[19] P. Novak, T. J. Jensen, J. C. Garbe, M. R. Stampfer, and B. W. Futscher, "Stepwise DNA methylation changes are linked to escape from defined proliferation barriers and mammary epithelial cell immortalization," Cancer Research, vol. 69, no. 12, pp. 5251-5258, 2009.

[20] J. Xu, P. B. Shetty, W. Feng et al., "Methylation of HIN-1, RASSF1A, RIL and CDH13 in breast cancer is associated with clinical characteristics, but only RASSF1A methylation is associated with outcome," BMC Cancer, vol. 12, no. 1, p. 243, 2012.

[21] M. S. Pepe, R. Etzioni, Z. Feng et al., "Phases of biomarker development for early detection of cancer," JNCI Journal of the National Cancer Institute, vol. 93, no. 14, pp. 1054-1061, 2001.

[22] S. E. Lim, M. Back, E. Quek, P. Iau, T. Putti, and J. E. L. Wong, "Clinical observations from a breast cancer registry in Asian women," World Journal of Surgery, vol. 31, no. 7, pp. 13871392, 2007.

[23] J. M. Harvey, G. M. Clark, C. K. Osborne, and D. C. Allred, "Estrogen receptor status by immunohistochemistry is superior to the ligand-binding assay for predicting response to adjuvant endocrine therapy in breast cancer," Journal of Clinical Oncology, vol. 17, no. 5, pp. 1474-1481, 1999.

[24] R. Soong, P. D. Robbins, B. R. Dix et al., "Concordance between $\mathrm{p} 53$ protein overexpression and gene mutation in a large series of common human carcinomas," Human Pathology, vol. 27, no. 10, pp. 1050-1055, 1996.

[25] L. C. Panasci, "Breast cancer subtypes and response to docetaxel in node-positive breast cancer: use of an immunohistochemical definition in the BCIRG 001 trial," Journal of Clinical Oncology, vol. 27, no. 26, article e111, 2009.

[26] C. A. Eads, K. D. Danenberg, K. Kawakami et al., "MethyLight: a high-throughput assay to measure DNA methylation," Nucleic Acids Research, vol. 28, no. 8, article e32, 2000.

[27] S. Ogino, T. Kawasaki, M. Brahmandam et al., "Precision and performance characteristics of bisulfite conversion and real- 
time PCR (MethyLight) for quantitative DNA methylation analysis," The Journal of Molecular Diagnostics, vol. 8, no. 2, pp. 209-217, 2006.

[28] M. Widschwendter, K. D. Siegmund, H. M. Müller et al., "Association of breast cancer DNA methylation profiles with hormone receptor status and response to tamoxifen," Cancer Research, vol. 64, no. 11, pp. 3807-3813, 2004.

[29] Y. H. Cho, J. Shen, M. D. Gammon et al., "Prognostic significance of gene-specific promoter hypermethylation in breast cancer patients," Breast Cancer Research and Treatment, vol. 131, no. 1, pp. 197-205, 2012.

[30] W. Wang and S. Srivastava, "Strategic approach to validating methylated genes as biomarkers for breast cancer," Cancer Prevention Research, vol. 3, no. 1, pp. 16-24, 2010.

[31] K. J. Livak and T. D. Schmittgen, "Analysis of relative gene expression data using real-time quantitative PCR and the $2^{-\Delta \Delta C}$ T method," Methods, vol. 25, no. 4, pp. 402-408, 2001.

[32] S. J. Clark and J. Melki, "DNA methylation and gene silencing in cancer: which is the guilty party?," Oncogene, vol. 21, no. 35, pp. 5380-5387, 2002.

[33] A. A. Muggerud, J. A. Rønneberg, F. Wärnberg et al., "Frequent aberrant DNA methylation of $A B C B 1, F O X C 1$, $P P P 2 R 2 B$ and $P T E N$ in ductal carcinoma in situ and early invasive breast cancer," Breast Cancer Research, vol. 12, no. 1, article R3, 2010.

[34] K. Kerlikowske, D. Grady, J. Barclay, E. A. Sickles, and V. Ernster, "Effect of age, breast density, and family history on the sensitivity of first screening mammography," JAMA, vol. 276, no. 1, pp. 33-38, 1996.

[35] A. Kwong, L. P. Wong, H. N. Wong et al., “A BRCA2 founder mutation and seven novel deleterious BRCA mutations in southern Chinese women with breast and ovarian cancer," Breast Cancer Research and Treatment, vol. 117, no. 3, pp. 683-686, 2009.

[36] J. Li, B. N. Zhang, J. H. Fan et al., “A nation-wide multicenter 10year (1999-2008) retrospective clinical epidemiological study of female breast cancer in China," BMC Cancer, vol. 11, no. 1, p. 364, 2011.

[37] G. N. Hortobagyi, J. de la Garza Salazar, K. Pritchard et al., "The global breast cancer burden: variations in epidemiology and survival," Clinical Breast Cancer, vol. 6, no. 5, pp. 391401, 2005.

[38] B. O. Anderson, R. Shyyan, A. Eniu et al., "Breast cancer in limited-resource countries: an overview of the Breast Health Global Initiative 2005 guidelines," The Breast Journal, vol. 12, Supplement 1, pp. S3-15, 2006.

[39] I. Saarenmaa, T. Salminen, U. Geiger et al., "The effect of age and density of the breast on the sensitivity of breast cancer diagnostic by mammography and ultasonography," Breast Cancer Research and Treatment, vol. 67, no. 2, pp. 117-123, 2001.

[40] K. Holm, C. Hegardt, J. Staaf et al., "Molecular subtypes of breast cancer are associated with characteristic DNA methylation patterns," Breast Cancer Research, vol. 12, no. 3, article R36, 2010.

[41] A. Petronis, "Epigenetics as a unifying principle in the aetiology of complex traits and diseases," Nature, vol. 465, no. 7299, pp. 721-727, 2010.

[42] L. van Neste, J. G. Herman, G. Otto, J. W. Bigley, J. I. Epstein, and W. van Criekinge, "The epigenetic promise for prostate cancer diagnosis," The Prostate, vol. 72, no. 11, pp. 1248 1261, 2012.

[43] S. S. K. Tang and G. P. H. Gui, "Biomarkers in the diagnosis of primary and recurrent breast cancer," Biomarkers in Medicine, vol. 6, no. 5, pp. 567-585, 2012.

[44] S. Karray-Chouayekh, F. Trifa, A. Khabir et al., "Aberrant methylation of RASSF1A is associated with poor survival in Tunisian breast cancer patients," Journal of Cancer Research and Clinical Oncology, vol. 136, no. 2, pp. 203-210, 2010.

[45] S. Wang, T. H. Dorsey, A. Terunuma, R. A. Kittles, S. Ambs, and B. Kwabi-Addo, "Relationship between tumor DNA methylation status and patient characteristics in AfricanAmerican and European-American women with breast cancer," PLoS One, vol. 7, no. 5, article e37928, 2012.

[46] H. Schwarzenbach, D. S. B. Hoon, and K. Pantel, "Cell-free nucleic acids as biomarkers in cancer patients," Nature Reviews Cancer, vol. 11, no. 6, pp. 426-437, 2011.

[47] H. C. Chiou, M. V. Tangco, K. Kormis et al., "Enhanced resistance to nuclease degradation of nucleic acids complexed to asialoglycoprotein-polylysine carriers," Nucleic Acids Res, vol. 22, no. 24, pp. 5439-5446, 1994.

[48] M. O. Hoque, Q. Feng, P. Toure et al., "Detection of aberrant methylation of four genes in plasma DNA for the detection of breast cancer," Journal of Clinical Oncology, vol. 24, no. 26, pp. 4262-4269, 2006.

[49] J. D. Brooks, P. Cairns, R. E. Shore et al., "DNA methylation in pre-diagnostic serum samples of breast cancer cases: results of a nested case-control study," Cancer Epidemiology, vol. 34, no. 6, pp. 717-723, 2010.

[50] R. A. Gupta, N. Shah, K. C. Wang et al., "Long non-coding RNA HOTAIR reprograms chromatin state to promote cancer metastasis," Nature, vol. 464, no. 7291, pp. 1071-1076, 2010.

[51] Y. Korshunova, R. K. Maloney, N. Lakey et al., "Massively parallel bisulphite pyrosequencing reveals the molecular complexity of breast cancer-associated cytosine-methylation patterns obtained from tissue and serum DNA," Genome Research, vol. 18, no. 1, pp. 19-29, 2008.

[52] R. Métivier, R. Gallais, C. Tiffoche et al., "Cyclical DNA methylation of a transcriptionally active promoter," Nature, vol. 452, no. 7183, pp. 45-50, 2008.

[53] S. Yegnasubramanian, M. C. Haffner, Y. Zhang et al., "DNA hypomethylation arises later in prostate cancer progression than CpG island hypermethylation and contributes to metastatic tumor heterogeneity," Cancer Research, vol. 68, no. 21, pp. 8954-8967, 2008.

[54] N. G. Bediaga, A. Acha-Sagredo, I. Guerra et al., "DNA methylation epigenotypes in breast cancer molecular subtypes," Breast Cancer Research, vol. 12, no. 5, article R77, 2010.

[55] S. Y. Park, H. J. Kwon, Y. Choi et al., "Distinct patterns of promoter CpG island methylation of breast cancer subtypes are associated with stem cell phenotypes," Modern Pathology, vol. 25, no. 2, pp. 185-196, 2012.

[56] N. Bloushtain-Qimron, J. Yao, E. L. Snyder et al., "Cell typespecific DNA methylation patterns in the human breast," Proceedings of the National Academy of Sciences of the United States of America, vol. 105, no. 37, pp. 14076-14081, 2008. 


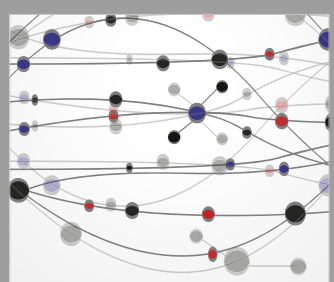

The Scientific World Journal
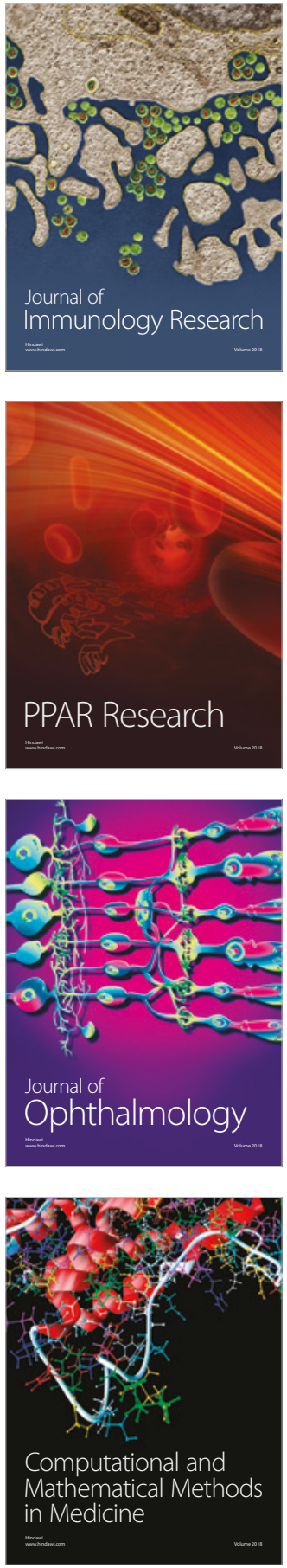

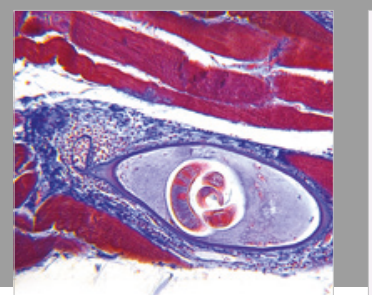

Gastroenterology Research and Practice

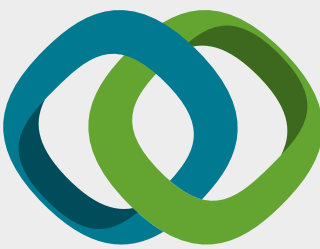

\section{Hindawi}

Submit your manuscripts at

www.hindawi.com
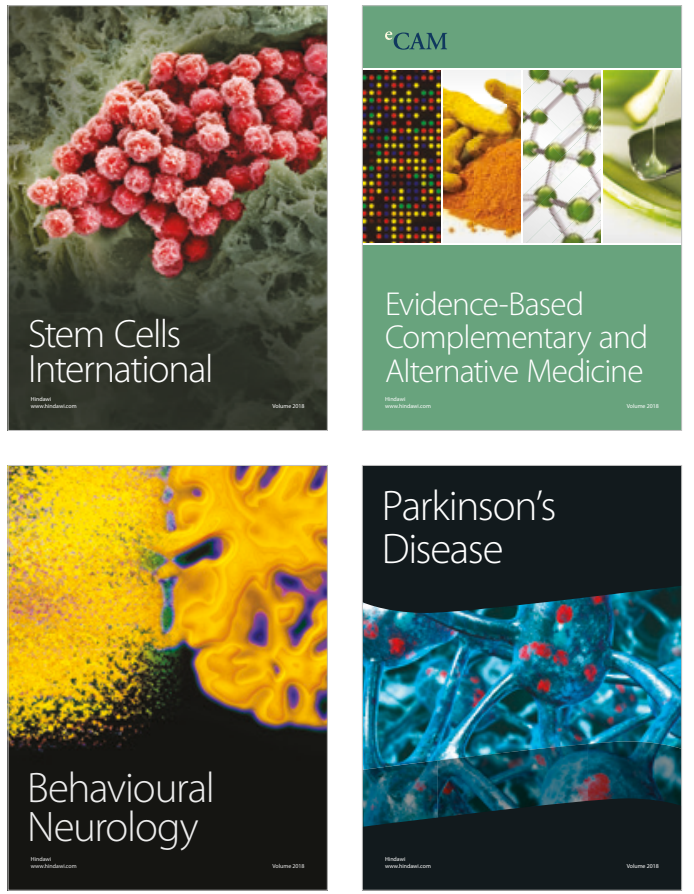

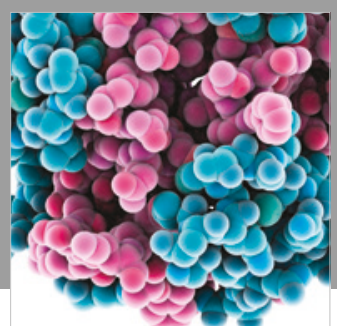

ournal of

Diabetes Research

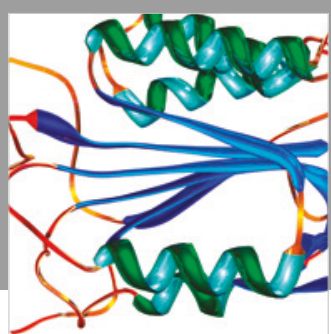

Disease Markers
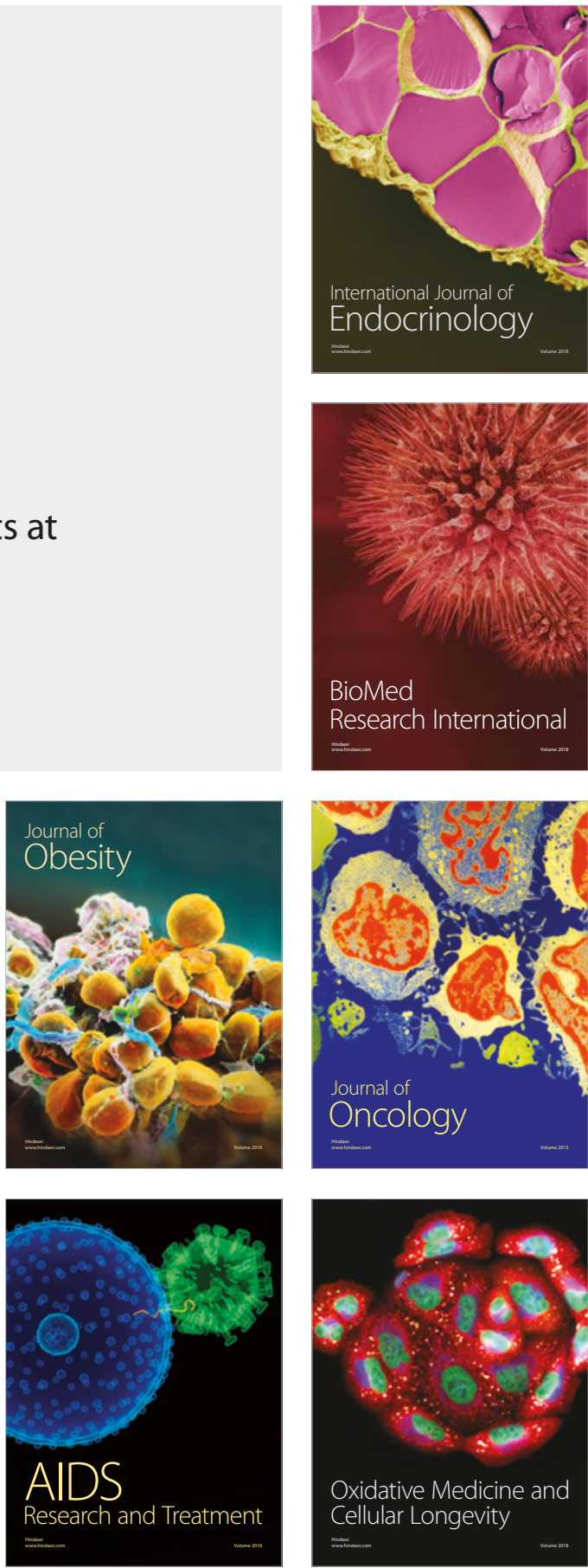